ANNALES

POLONICI MATHEMATICI

$84.1(2004)$

\title{
Non-special projectively normal line bundles on general $k$-gonal curves
}

\author{
by E. BALlico (Trento)
}

\begin{abstract}
Let $X$ be a sufficiently general smooth $k$-gonal curve of genus $g$ and $R \in$ $\operatorname{Pic}(X)$ the degree $k$ spanned line bundle. We find an optimal integer $z>0$ such that the line bundle $R^{\otimes z}$ is very ample and projectively normal.
\end{abstract}

1. Introduction. Let $X$ be a smooth and connected projective curve of genus $g$, and $L$ a spanned line bundle on $X$. Let $h_{L}: X \rightarrow \mathbb{P}\left(H^{0}(X, L)^{*}\right)$ denote the morphism induced by the complete linear system $|L|$. Here we assume that $X$ is a sufficiently general $k$-gonal curve and explore the projective normality of non-special very ample line bundles on $X$.

Our main result is the following theorem giving the projective normality of several non-special line bundles $L$ on general $k$-gonal curves of genus $g$. Indeed, often $\operatorname{deg}(L) \ll 3 g / 2$ and hence this is outside the range covered in [4]. The result (and its proof) is very different from the case of special line bundles considered in [6] (see in particular lines 4-8 of page 189 of [6]) and the references therein.

Theorem 1. Fix integers $k \geq 3, g \geq 3 k$ and $y \geq 1+\lceil g /(k-1)\rceil$. Let $X$ be a smooth and connected $k$-gonal curve. Let $R \in \operatorname{Pic}^{k}(X)$ be any line bundle computing the gonality of $X$. Assume that the scrollar invariants of the pair $(X, R)$ are the ones of a general $k$-gonal curve of genus $g$, i.e. $h^{0}\left(X, R^{\otimes t}\right)=t+1$ for $0 \leq t \leq\left[g([k-1)]\right.$ and $h^{1}\left(X, R^{\otimes t}\right)=0$ for $t>$ $[g /(k-1)]$ (see [1] in arbitrary characteristic or [3, 1.1], [2, Prop. 1.1] when $\operatorname{char}(\mathbb{K})=0)$. Set $L:=R^{\otimes y}$. Then $L$ is very ample and projectively normal.

Take $(X, R)$ as in the statement of Theorem 1 . If $g /(k-1) \in \mathbb{Z}$, then $h^{0}\left(X, R^{\otimes(g /(k-1))}\right)=1+g /(k-1)$ and $h_{R^{\otimes(g /(k-1)}}(X)$ is a rational normal

2000 Mathematics Subject Classification: Primary 14H51.

Key words and phrases: line bundles on curves, vector bundles on curves, plane curves, nodal plane curves.

The author was partially supported by MIUR and GNSAGA of INdAM (Italy). 
curve in $\mathbb{P}^{g /(k-1)}$. Hence Theorem 1 is sharp in this case. From Theorem 1 we will easily obtain the following result.

Corollary 1 . Let $X$ be a general $k$-gonal curve of genus $g \geq 2 k+1$. Then for every integer $d \geq k\lceil g /(k-1)\rceil+k$ a general $L \in \operatorname{Pic}^{d}(X)$ is projectively normal.

REMARK 1 . Let $X$ be a general $k$-gonal curve of genus $g$. If $k \approx g / 4$, then Corollary 1 covers approximately the range $d \gtrsim 5 g / 4+5$. If $g \geq 2 k+2$, then every smooth $k$-gonal curve of genus $g$ is a flat limit of a flat family of $(k+1)$-gonal curves. Hence by semicontinuity if $k \geq g / 4$, then on $X$ we roughly cover the range $d \gtrsim 5 g / 4+5$.

Then we will explore another case and prove the following result.

Proposition 1. Fix integers $z \geq 3$ and $k \geq 3$. Let $X$ be a smooth and connected projective curve of genus $g$ and $R \in \operatorname{Pic}^{k}(X)$, and set $L:=R^{\otimes z}$. Assume $R$ is spanned, $h^{0}\left(X, R^{\otimes(z-1)}\right)=z$ and $h^{0}\left(X, R^{\otimes z}\right)=z+2$. Then $z(k-1)-1 \leq g \leq 1+k(k z-z-2) / 2$. The morphism $h_{L}$ is birational onto its image. $L$ is very ample if and only if $g=1+k(k z-z-2) / 2$. We have $g=z(k-1)-1$ if and only if the pair $(X, R)$ has the same scrollar invariants as a general $k$-gonal curve of genus $g$. Set $Y:=h_{L}(X)$. Then $p_{a}(Y)=1+k(k z-z-2) / 2$ and the degree $z k$ curve $Y \subset \mathbb{P}^{z+1}$ is the complete intersection of a cone $T$ over a rational normal curve in $\mathbb{P}^{z}$ with a degree $k$ hypersurface. We have $h^{1}\left(\mathbb{P}^{z+1}, \mathcal{I}_{Y}(t)\right)=0$ for every $t \in \mathbb{Z}$.

\section{The proofs}

Proof of Theorem 1. To fix the notation we divide the proof in two parts: in part (i) we assume $g /(k-1) \in \mathbb{Z}$, while in part (ii) we assume $g /(k-1) \notin \mathbb{Z}$.

(i) Here we assume $g /(k-1) \in \mathbb{Z}$. We will first check that $L:=R^{\otimes y}$ is very ample, which is equivalent to $h^{0}(X, L(-Z))=h^{0}(X, L)-2$ for every degree two zero-dimensional scheme $Z \subset X$. Since $R$ is spanned, this equality is obvious if $Z$ is not contained in a fiber of the degree $k$ pencil $h_{R}: X \rightarrow \mathbb{P}^{1}$. Assume that $Z$ is contained in such a fiber, say $Z+B \in|R|$ with $B \geq 0$. Assume $h^{0}(X, L(-Z)) \neq h^{0}(X, L)-2$, i.e. $h^{1}(X, L(-Z))>h^{1}(X, L)=0$. Since $L(-Z) \cong R^{\otimes x}(B)$ we obtain $h^{1}\left(X, R^{\otimes x}(B)\right)>0$ and hence $h^{1}\left(X, R^{\otimes x}\right)>0$, a contradiction.

Let $u: X \rightarrow \mathbb{P}^{k y-g}$ be the embedding associated to the complete linear system $\left|R^{\otimes y}\right|$. Fix $y$ general divisors $D_{i} \in|R|, 1 \leq i \leq y$, say $D_{i}=P_{i, 1}+$ $\cdots+P_{i, k}$, and let $A_{i}:=\left\langle\left\{u\left(P_{i, 1}\right), \ldots, u\left(P_{i, k}\right)\right\}\right\rangle$ denote the linear span of the set $\left\{u\left(P_{i, 1}\right), \ldots, u\left(P_{i, k}\right)\right\}$. Since $h^{1}\left(X, R^{\otimes(y-1)}\right)=0$, we have $\operatorname{dim}\left(A_{i}\right)=k-1$ for every $i$. Since each set $A_{i} \cap u(X)$ is finite and the divisors $D_{1}, \ldots, D_{m}$ are general, and $R$ has no base point, we may always assume $u\left(P_{h, j}\right) \notin A_{i}$ for 
all $i, h, j$ such that $i \neq h$. Thus $\operatorname{dim}\left(\left\langle A_{i} \cup\left\{P_{h, j}\right\}\right\rangle\right)=\operatorname{dim}\left(A_{i}\right)+1=k$ for all $i, h, j$ such that $i \neq h$. Similarly for any $I \subsetneq\{1, \ldots, y\}$, any $h \in\{1, \ldots, y\}$ and any $j, 1 \leq j \leq k$, we may assume $u\left(P_{h, j}\right) \notin\left\langle\bigcup_{i \in I} A_{i}\right\rangle$.

First assume $y=g /(k-1)+1$. Since $h^{0}\left(X, L \otimes\left(R^{\otimes 2}\right)^{*}\right)=h^{0}(X, L)-k-1$ in this case, we have $\operatorname{dim}\left(\left\langle A_{y} \cup A_{y-1}\right\rangle\right)=k+1$ and hence $\left\langle A_{y} \cup\left\{u\left(P_{y-1, j}\right)\right\}\right\rangle=$ $\left\langle A_{y} \cup A_{y-1}\right\rangle$. Since $u\left(P_{y, j}\right) \notin A_{y-1}$ and $u\left(P_{y-1, j}\right) \notin A_{y}$ for all $j$, the points $u\left(P_{i, j}\right), i \in\{y-1, y\}, 1 \leq j \leq k$, are in linearly general position in the projective space $\left\langle A_{y} \cup A_{y-1}\right\rangle$. By [5, Th. 2.1], the set $\left\{u\left(P_{i, j}\right)\right\}, i \in\{y-1, y\}$, $1 \leq j \leq k$, has Properties $N_{0}$ and $N_{1}$; we will only use Property $N_{0}$. Since $L$ is very ample and non-special, to check that it has Property $N_{0}$ (i.e. it is projectively normal) it is sufficient to find a hyperplane section of $u(X)$ with Property $N_{0}$. First we will check that the union $S$ of the points $P_{i, j}$, $y-2 \leq i \leq y, 1 \leq j \leq k$, has Property $N_{0}$, i.e. it imposes $3 k$ independent conditions on the quadric hypersurfaces containing them. This statement is equivalent to the same statement in the linear span $\left\langle A_{y-2} \cup A_{y-1} \cup A_{y}\right\rangle$. Now, $\left\langle A_{y-1} \cup A_{y}\right\rangle$ is a hyperplane in $\left\langle A_{y-2} \cup A_{y-1} \cup A_{y}\right\rangle$ containing $2 k$ points of $S$ with property $N_{0}$. Set $S^{\prime}:=S \cap\left\langle A_{y-1} \cup A_{y}\right\rangle$. Notice that the points $u\left(P_{y-2, j}\right)$, $1 \leq j \leq k$, are in linearly general position. Hence for every $j, 1 \leq j \leq k$, there is a hyperplane $H_{j}$ in $\left\langle A_{y-2} \cup A_{y-1} \cup A_{y}\right\rangle$ containing all $P_{y-2, h}, h \neq j$, but not $P_{y-2, j}$. The reducible quadric hypersurfaces $H_{j} \cup\left\langle A_{y-1} \cup A_{y}\right\rangle, 1 \leq$ $j \leq k$, show that $S \backslash S^{\prime}$ gives $k$ independent conditions on the linear space of all quadric hypersurfaces containing $S^{\prime}$. Thus $S$ imposes $3 k$ independent conditions on quadrics, i.e. it has Property $N_{0}$.

Now assume $y \geq g /(k-1)+2$. Since $h^{1}\left(X, R^{\otimes(g /(k-1))}\right)=0$ and $h^{0}\left(X, R^{\otimes(g /(k-1))}\right)=h^{0}\left(X, R^{\otimes(g /(k-1)+1)}\right)$, we have $\operatorname{dim}\left(\left\langle\bigcup_{i=g /(k-1)+1}^{y} A_{i}\right\rangle\right)$ $=(y-g /(k-1)) k-1$ and $\operatorname{dim}\left(\left\langle\bigcup_{i=g /(k-1)}^{y} A_{i}\right\rangle\right)=(y-g /(k-1)) k$. Follow step by step the previous proof using $\left\langle\bigcup_{i=g /(k-1)}^{y} A_{i}\right\rangle$ instead of $A_{y}$.

(ii) Now we assume $g /(k-1) \notin \mathbb{Z}$. First, we assume $y=\lceil g((k-1)\rceil$. In this case we have

$$
\begin{aligned}
h^{0}\left(X, L \otimes\left(R^{*}\right)^{\otimes 2}\right) & =h^{0}(X, L)-k-1+g-(k-1)\lceil g /(k-1)\rceil \\
& <h^{0}(X, L)-k-1
\end{aligned}
$$

and hence $A_{y}$ is not a hyperplane in $\left\langle A_{y}+A_{y-1}\right\rangle$. However, $\left\langle A_{y}+A_{y-1}\right\rangle \cap$ $u(X)$ contains again exactly $2 k$ points in linearly independent position and hence the proof of part (i) works with only trivial modifications. The case $y>\lceil g((k-1)\rceil$ is done step by step as in part (i) without any modification.

Lemma 1. Let $X$ be a smooth and connected projective curve, $A \in$ $\operatorname{Pic}(X)$ such that $h^{1}(X, A)=0$, and $P \in X$. Then $h^{1}(X, A(P))=0$. If $A$ is very ample, then $A(P)$ is very ample. If $A$ is very ample and projectively normal, then $A(P)$ is very ample and projectively normal. 
Proof. Obviously, $h^{1}(X, A(P)) \leq h^{1}(X, A)=0$. A line bundle $M$ on $X$ is very ample if and only if for every effective degree two divisor $Z$ on $X$ we have $h^{0}(X, M(-Z))=h^{0}(X, M)-2$, i.e. $h^{1}(X, M(-Z))=h^{1}(X, M)$. Fix an effective degree two divisor $Z$ of $X$. Assume that $A$ is very ample. Hence $h^{1}(X, A(-Z))=h^{1}(X, A)=0$. Thus $h^{1}(X, A(P)(-Z))=0$, proving the very ampleness of $A(P)$.

Let $\mu_{A}: S^{2}\left(H^{0}(X, A)\right) \rightarrow H^{0}\left(X, A^{\otimes 2}\right)$ and $\mu_{A(P)}: S^{2}\left(H^{0}(X, A(P))\right) \rightarrow$ $H^{0}\left(X, A^{\otimes 2}(2 P)\right)$ be the multiplication maps. Assume $A$ is projectively normal. Thus $\mu_{A}$ is surjective. Since $h^{1}(X, A(P))=0$, Castelnuovo-Mumford's lemma says that to prove that $A(P)$ is projectively normal it is sufficient to check the surjectivity of $\mu_{A(P)}$. The choice of the point $P$ allows us to view $H^{0}(X, A)$ as the hyperplane of $H^{0}(X, A(P))$ formed by all sections vanishing at $P$, and $H^{0}\left(X, A^{\otimes 2}\right)\left(\right.$ resp. $\left.H^{0}\left(X, A^{\otimes 2}(P)\right)\right)$ as the codimension two (resp. one) linear subspace of $H^{0}\left(X, A^{\otimes 2}(2 P)\right)$ formed by the sections vanishing at $P$ at least to order two (resp. one). The surjectivity of $\mu_{A}$ implies that $\mu_{A(P)}$ contains $H^{0}\left(X, A^{\otimes 2}\right)$. Since $A$ is spanned and $h^{0}(X, A(P))=h^{0}(X, A)+1$, the multiplication map $H^{0}(X, A) \otimes H^{0}(X, A(P)) \rightarrow H^{0}\left(X, A^{\otimes 2}(P)\right)$ contains a section not vanishing at $P$ (as an element of $H^{0}\left(X, A^{\otimes 2}(P)\right)$ ), i.e. an element of $H^{0}\left(X, A^{\otimes 2}(2 P)\right)$ with exact order one at $P$. The spannedness of $A(P)$ implies that $\operatorname{Im}\left(\mu_{A(P)}\right)$ contains an element not vanishing at $P$. Hence $\operatorname{Im}\left(\mu_{A}\right)$ has codimension at least two in $\operatorname{Im}\left(\mu_{A(P)}\right)$, proving that $\operatorname{Im}\left(\mu_{A(P)}\right)=H^{0}\left(X, A^{\otimes 2}(2 P)\right)$.

Proof of Corollary 1. Let $R \in \operatorname{Pic}^{k}(X)$ be the line bundle computing the gonality of $X$. Since $d \geq g+3$, a general $L \in \operatorname{Pic}^{d}(X)$ is non-special and very ample. By semicontinuity it is sufficient (for any fixed $d$ ) to find one non-special, very ample and projectively normal degree $d$ line bundle on $X$. If $d=k\lceil g /(k-1)\rceil+k$, then take $L:=R^{\otimes(d / k)}$. If $d>k\lceil g /(k-1)\rceil+k$, then apply Lemma $1 d-k\lceil g /(k-1)\rceil-k$ times.

Proof of Proposition 1. Since $h^{0}\left(X, R^{\otimes(z-1)}\right)=z$ and $R$ is spanned, for $1 \leq j \leq z-1$ we have $h^{0}\left(X, R^{\otimes j}\right)=j+1, R^{\otimes j}$ is spanned, the morphism $h_{R^{\otimes j}}(X)$ is a rational normal curve in $\mathbb{P}^{j}$ and the morphism $h_{R^{\otimes j}}$ has degree $k$ onto its image. As $h^{0}(X, L)=z+2$ and $\operatorname{deg}(L)=z k$, we have $g \leq z(k-1)$ -1 by Riemann's inequality. Since $h^{0}(X, L)>z+1$, the curve $Y:=h_{L}(X)$ is not a rational normal curve of $\mathbb{P}^{z+1}$. Since $h^{0}\left(X, L \otimes R^{*}\right)=h^{0}(X, L)-2$, the scheme $h_{L}(D)$ spans a line $\left\langle h_{L}(D)\right\rangle$ for every effective divisor $D \in|R|$. Since $h^{0}\left(X, L \otimes R^{*} \otimes R^{*}\right)=h^{0}\left(X, L \otimes R^{*}\right)-1$, we have $\left\langle h_{L}(D)\right\rangle \cap\left\langle h_{L}\left(D^{\prime}\right)\right\rangle \neq \emptyset$ for all $D, D^{\prime} \in|R|$. It is easy to check that for any infinite algebraic family $\left\{D_{\gamma}\right\}_{\gamma \in \Gamma}$ of mutually intersecting lines in $\mathbb{P}^{z+1}$ parametrized by an integral variety $\Gamma$ either there is a plane $A$ containing $\bigcup_{\gamma \in \Gamma} D_{\gamma}$ or there is $Q \in \bigcap_{\gamma \in \Gamma} D_{\gamma}$. In our set-up with the lines $\left\langle h_{L}(D)\right\rangle, D \in|R|$, the first case cannot occur 
because $Y=h_{L}(X)$ is non-degenerate. Hence there is a point $Q$ common to all these lines. The projection, $C$, of $Y$ from $Q$ is a non-degenerate curve in $\mathbb{P}^{z}$ of degree at most $z$ and with degree $z$ only if $Q \notin Y$ and $h_{L}$ is birational onto its image $Y$. Hence $C$ is a rational normal curve in $\mathbb{P}^{z}, Q \notin Y$ and $h_{L}: X \rightarrow Y$ is birational. Hence $p_{a}(Y) \geq g$ and $p_{a}(Y)=g$ if and only if $L$ is very ample.

The curve $Y$ is contained in the cone $T$ with base $C$ and vertex $Q$. Let $\phi: S \rightarrow T$ be the blowing-up of $Q$. Set $h:=\phi^{-1}(Q)$. It is well known that $S$ isomorphic to the Hirzebruch surface $F_{z}$ and that $h$ is the section of the ruling $v: S \rightarrow \mathbb{P}^{1}$ with negative self-intersection. Let $f$ denote any fiber of the ruling $v$. We have $\operatorname{Pic}(Z) \cong \mathbb{Z} h \oplus \mathbb{Z} f, h^{2}=-z, h \cdot f=1$ and $f^{2}=0$. Let $Y^{\prime} \subset S$ be the strict transform of $Y$. Since $Q \notin Y$, we have $Y^{\prime} \cap h=\emptyset$. Thus $Y^{\prime} \cdot h=0$. Since $Y^{\prime} \cdot f=k$, we obtain $Y^{\prime} \in|k h+k z f|$. We have $\omega_{S} \cong \mathcal{O}_{S}(-2 h-(z+2) f)$. Thus $\omega_{Y^{\prime}} \cong \mathcal{O}_{Y^{\prime}}((k-2) h+(k z-z-2) f)$. Hence $p_{a}\left(Y^{\prime}\right)=1+k(k z-z-2) / 2$. Since $Q \notin Y$, we have $Y^{\prime} \cong Y$ and hence $p_{a}(Y)=1+k(k z-z-2) / 2$.

Claim. We have $h^{1}\left(\mathbb{P}^{z+1}, \mathcal{I}_{T}(x)\right)=h^{1}\left(T, \mathcal{O}_{T}(x)\right)=0$ for every $x \in \mathbb{Z}$.

Proof of the Claim. Since $C$ is arithmetically Cohen-Macaulay in $\mathbb{P}^{z}$, it follows that $T$ is arithmetically Cohen-Macaulay in $\mathbb{P}^{z+1}$, proving the Claim.

The natural map $H^{0}\left(T, \mathcal{O}_{T}(k)\right) \rightarrow H^{0}\left(S, \mathcal{O}_{S}(k h+k z f)\right)$ is surjective. Since $Y^{\prime} \in|k h+k z f|$, the second vanishing of the Claim for the integer $x:=k$ implies that $Y$ is scheme-theoretically the intersection of $T$ with a degree $k$ hypersurface. By the first vanishing of the Claim for every integer $t>0$ the restriction map $H^{0}\left(\mathbb{P}^{z+1}, \mathcal{O}_{\mathbb{P}^{z+1}}(t)\right) \rightarrow H^{0}\left(T, \mathcal{O}_{T}(t)\right)$ is surjective. Since $Y$ is scheme-theoretically the zero-locus of an element of $H^{0}\left(X, \mathcal{O}_{T}(k)\right)$, the surjectivity of the restriction map $H^{0}\left(T, \mathcal{O}_{T}(t)\right) \rightarrow$ $H^{0}\left(Y, \mathcal{O}_{Y}(t)\right)$ follows from the second vanishing of the Claim for the integer $x:=t-k$.

\section{References}

[1] E. Ballico, A remark on linear series on general k-gonal curves, Boll. Un. Mat. Ital. A (7) (1989), 195-197.

[2] M. Coppens, C. Keem and G. Martens, The primitive length of a general k-gonal curve, Indag. Math. 5 (1994), 145-159.

[3] M. Coppens and G. Martens, Linear series on a general k-gonal curve, Abh. Math. Sem. Univ. Hamburg 69 (1999), 347-371.

[4] M. Green and R. Lazarsfeld, On the projective normality of complete linear series on an algebraic curve, Invent. Math. 83 (1986), 73-90.

[5] - - - Some results on the syzygies of finite sets and algebraic curves, Compositio Math. 67 (1988), 301-314. 
[6] S. Kim and Y. R. Kim, Projectively normal embedding of a k-gonal curve, Comm. Algebra 32 (2004), 187-201.

Department of Mathematics

University of Trento

38050 Povo (TN), Italy

E-mail: ballico@science.unitn.it

Reçu par la Rédaction le 15.4.2004 